REVIEW

\title{
Management of thyroid disorders
}

\author{
L D K E Premawardhana, J H Lazarus
}

Postgrad Med J 2006;82:552-558. doi: 10.1136/pgmj.2006.047290

Autoimmune thyroid disease is the predominant form of thyroid dysfunction in the developed world. Although its precise cause is currently unclear, principles of management have been established. There is a vigorous debate about the management of the increasingly commonly recognised subclinical forms of thyroid dysfunction despite recent recommendations. Nodular thyroid disease and thyroid carcinoma have received wide attention. The effects of drugs and pregnancy on thyroid function have also been investigated widely. This short review attempts to give an overview and clarify the current management of common thyroid disorders.

See end of article for authors' affiliations ......................

Correspondence to: DrLDKE Premawardhana, Department of Medicine, Caerphilly Miners' Hospital, St Martin's Road, Caerphilly CF83 2WW UK; Ldke.

Premawardhana@gwent. wales.nhs.uk

Submitted

28 February 2006

Accepted 10 April 2006
$\mathrm{T}$ he commonest cause of thyroid disease worldwide is iodine deficiency, which causes goitre and hypothyroidism in some. However, autoimmune thyroid disease is the predominant form of thyroid dysfunction in the developed world. Although genetic (HLA-DR3, CTLA-4, and thyroglobulin gene mutations) and environmental factors (infection, smoking, iodine status) have been implicated, its precise cause is unclear. ${ }^{12}$

Overt thyrotoxicosis or hypothyroidism occurs in about $2 \%$ of women and $0.2 \%$ of men in the UK. $^{3}$ The incidence of hypothyroidism and hyperthyroidism in women is $4.1 / 1000$ and 0.8 / 1000 per year respectively, but is much lower in men for both $(0.6 / 1000$ per year $) .{ }^{4}$ More recent data suggest a higher incidence, for example, 4.98 (women) and 0.88/1000/year (men) for hypothyroidism and 0.77 (women) and 0.14 / 1000/year (men) for hyperthyroidism. ${ }^{5}$ The incidence of overt thyroid dysfunction may depend on population iodine intake. ${ }^{6}$ The main causes of hypothyroidism in the developed world are Hashimoto's disease, and thyroid ablation (radioactive iodine, surgery, and drugs); and of thyrotoxicosis are Graves' disease (GD) in 70\%, toxic multinodular goitre (TMNG), and toxic adenoma (TA). Subclinical thyroid dysfunction tion) is diagnosed more often because of widespread thyroid testing in modern clinical practice. Clear management strategies are however yet to emerge.

We describe briefly the management of common thyroid disorders highlighting recent advances and unresolved issues. A comprehensive account of pathophysiology, clinical features, and investigations in all cases is not within the scope of this review. (commonly in women and the elderly popula-

\section{HYPOTHYROIDISM}

Permanent hypothyroidism, commonly attributable to Hashimoto's thyroiditis (and its fibrotic variant atrophic thyroiditis), requires lifelong thyroxine (LT4) treatment. However, reversible hypothyroidism requiring only short term treatment needs to be identified (box 1). ${ }^{7}$

Management principles are clear in most (box 2 ), but the following points need to be noted:

(1) Therapeutic goals-particularly "normalisation" of thyroid stimulating hormone (TSH) activity. TSH targets have been revised down on the basis of most "normal" people having a TSH between $0.5-2.5 \mathrm{mU} / \mathrm{l}$ and of a higher percentage of unaffected people with TSH $>2 \mathrm{mU} / \mathrm{l}$ progressing to hypothyroidism. ${ }^{8}$ Most authorities now recommend 0.5$2.5 \mathrm{mU} / \mathrm{l}$ as the TSH target during replacement therapy.

(2) Combination therapy-there is no current evidence to support combination therapy of LT4 and liothyronine.'

(3) Bioequivalence of commercial preparations-American experts objected to recommendations for cost based substitution of LT4 preparations. ${ }^{10}$ The variable bioavailability of alternate preparations may adversely affect those with TSH activity at either end of the reference range and those whose replacement should be consistently to target (for example, pregnant women).

Replacement doses are higher in those with severe Hashimoto's compared with post-surgical and RAI therapy.

There are several causes for persistently raised TSH activity in patients receiving standard replacement doses of LT4 (box 3).

\section{GRAVES' DISEASE}

Antithyroid drugs (ATD), radioactive iodine (RAI) therapy, and surgery may be used alone or in combination during a first episode of Graves' disease. Most authorities recommend ATD as first line therapy in suitable patients in preparation for subsequent RAI or surgery, ${ }^{11} 12$ although ATD may also be used as sole long term

Abbreviations: GD, Graves' disease; TA, toxic adenoma; TMNG, toxic multinodular goitre; RAI, radioactive iodine; ATD, antithyroid drug; CMZ, carbimazole; PTU, propylthiouracil; MMI, methimazole; RCT, randomised controlled trial; TED, thyroid eye disease; TSH, thyroid stimulating hormone; PPTD, postpartum thyroid dysfunction; $\mathrm{SH}$, subclinical hypothyroidism; SHyper, subclinical hyperthyroidism; NTIS, non-thyroidal illness syndrome; HCG, human chorionic gonadotrophin 


\section{Box 1 Reversible causes of hypothyroidism}

- Hashimoto's thyroiditis (about 5\%)-decreasing thyroid stimulating hormone receptor blocking antibodies

- Postpartum thyroiditis - up to $70 \%$ become euthyroid in the first year

- Subacute thyroiditis - nearly $100 \%$ become euthyroid

- lodine induced-most become normal when iodine is withdrawn

- Drug induced-most recover when drugs are withdrawn

- Post-ablative (surgery/RAl therapy)-transient hypothyroidism occurs in some

therapy in those unwilling or unable to have definitive therapy (table 1).

\section{Antithyroid drugs}

CMZ and PTU are commonly used thionamide ATD (table 1). Both are easily and rapidly absorbed and have a long half life. Both drugs inhibit iodination of tyrosine in thyroglobulin but PTU also prevents conversion of thyroxine (T4) to triiodothyronine (T3) in thyroid and peripheral tissues-of advantage in severe thyrotoxicosis or thyrotoxic storm. Thionamides may also have immune suppressive effects. ${ }^{1}$

Despite randomised controlled trials (RCTs) examining individual drugs, drug regimens (ATD \pm thyroxine), and duration of therapy, there is as yet no clear consensus. ${ }^{13}$ Often decisions are based on personal preference and experience. A recent review of RCTs provides some clarity, and highlights the fact that high drop out and relapse rates with the many regimens of ATD ( \pm thyroxine) make definitive recommendations difficult. ${ }^{14}$ However, the titration regimen (significantly less side effects) for a period of 12-18 months seemed to have the benefit of evidence in its favour and is recommended as first line therapy. Early discussion of RAI therapy is also recommended in view of the high relapse rates with both ATD regimens.

\section{Follow up and remission}

Thyroid tests are done every four to six weeks (empirically) until clinical and biochemical euthyroidism is achieved and less commonly thereafter. Suppressed TSH activity may take a variable but long time to normalise despite normal or low free T4 activity.

There are no reliable predictors of remission or relapse despite several retrospective and one large prospective study. ${ }^{11}$ Large goitres, severe thyrotoxicosis, high freeT3/T4 ratios, and high baseline TRAb activity may predict low remission rates. The presence of TRAb at the end of ATD therapy predicts relapse ${ }^{15}$ although almost $30 \%-50 \%$ of TRAb negative persons also relapse. ${ }^{16}$ The benefit of these parameters is therefore doubtful in clinical practice.

\section{Side effects}

Minor side effects including skin reactions, arthralgias (sometimes heralding severe transient migratory polyarthritis), and gastrointestinal upsets occurs in about 5\%. Skin eruptions may require antihistamines while the ATD is continued or changed to a different group (cross reactivity occurring in about 50\%). Severe reactions may require stopping ATD and early RAI therapy.

The most feared side effect of ATD, agranulocytosis (a granulocyte count of less than $500 / \mathrm{cu} \mathrm{ml}$ ) is fortunately rare ( $0.37 \%$ for PTU and $0.35 \%$ for MMI) and is probably immune mediated. It usually occurs in the first three months, but late
Box 2 Principles of thyroxine replacement

therapy

- Start with $25-50 \mu \mathrm{g} /$ day of LT4 in the elderly and cardiac patients

- Start with 50-100 $\mu \mathrm{g} /$ day of LT4 in the young and healthy older patient

- Increase dose 4-6 weekly-aim for TSH "normalisation" and symptom control

- Corticosteroid replacement should be started before LT4 in those with suspected hypoadrenalism

- Higher doses may be required in gluten sensitivity, pregnancy, and concomitant drug therapy (box 3)

- Clinical and biochemical monitoring should be done every 6-12 months

presentation and first occurrence in a second course of ATD is known. A white cell count is recommended at start of therapy. There is no consensus about periodic neutrophil estimation as a predictor of agranulocytosis. Fever and sore throat are common presenting symptoms of agranulocytosis and patients should be warned to seek urgent medical attention. Withdrawal of ATD and appropriate inhospital intravenous antibiotic therapy (to include cover for pseudomonas) should be started immediately. G-CSF therapy and bone marrow aspiration should be guided by haematologists. The management of thyrotoxicosis in these patients may require unconventional modes of therapy and should be guided by thyroid specialists.

PTU may rarely cause an allergic hepatitis with a poor prognosis. Withdrawal of PTU and management of liver failure should be done in a specialised unit. This type of liver dysfunction should be differentiated from the more common, benign, and transient liver enzyme increase (up to six times normal) that is self limiting despite continued therapy. CMZ and MMI may cause cholestatic liver dysfunction that recovers on drug withdrawal. In the above situations the careful introduction of the alternative class of ATD or other drugs (for example, lithium) may have to be considered.

\section{Box 3 Raised TSH activity in patients receiving} standard replacement doses of LT4

- Non-compliance-supervised administration of standard daily or single weekly dose of $1000 \mu \mathrm{g}$

- Inadequate dose-dispensing error, change in formulation

- Interaction with drugs

- reduced absorption-iron tablets, cholestyramine, calcium carbonate, soya

- rapid clearance of LT4-phenytoin, carbamazepine, rifampicin, valproate

- Residual gland dysfunction

- Autoimmune, post-irradiation, surgery

- Pregnancy

- Postmenopausal oestrogen treatment (increase in TBG concentrations)

- Systemic illness 
Table 1 Antithyroid drugs in the management of Graves' disease

\begin{tabular}{ll}
\hline Drug & Regimen \\
\hline Carbimazole (CMZ), methimazole (MMI), & (1) "Titration regimen" - \\
propylthiouracil (PTU) - (written warning or & Start CMZ $40 \mathrm{mg} /$ day (or equivalent doses of MMI or PTU) \\
recorded verbal instructions about serious side & Reduce gradually to maintain euthyroidism (for example, \\
effects should be issued to all patients) & CMZ 5-10 mg/day) \\
& Continue for 12-18 months \\
& (2) "Block and replacement regimen" - \\
& Start CMZ $40 \mathrm{mg} /$ day or PTU 150 mg thrice daily \\
& Add thyroxine 100-150 mcg/day when euthyroid (at \\
& about 3-6 weeks) \\
& Continue for 6-9 months \\
& $20-80 \mathrm{mg}$ thrice daily to block adrenergic effects in the \\
initial few weeks
\end{tabular}

CMZ seems to have a slightly better side effect profile. With the titration regimen a 12-18 month course of therapy seems better (less relapses) than shorter or longer courses. A six month block and replacement regimen seems to be no less effective than a 12 month course. Recurrences are commonest in the first year after stopping treatment. ${ }^{11}$

ATD particularly PTU may cause vasculitis (some ANCA positive) with multisystem involvement. Immediate drug withdrawal (with remission in most), high dose glucocorticoids, cyclophosphamide, and haemodialysis may be required.

\section{RAl therapy}

RAI therapy is used increasingly as first line therapy for a first episode of GD after adolescence. Most recommend RAI as treatment of choice for recurrent disease and in the elderly population. RAI may be used as definitive therapy in those with severe heart, lung, or kidney disease contraindicating surgery. Contraindications to RAI include pregnancy, lactation, doubtful thyroid malignancy, and younger patients (for example, below 15 years). The presence of thyroid eye disease is a relative contraindication and is discussed below. Pregnancy should be avoided for four to six months after RAI therapy. About 5\%-20\% require a second dose (usually male, with severe disease and medium to large goitres); 10\%$20 \%$ become hypothyroid in the first year and 5\%-10\% per year thereafter. The incidence of hypothyroidism depends on the dose of RAI given-10\% are hypothyroid, $60 \%$ euthyroid, and $30 \%$ still thyrotoxic at one year; and $60 \%$ are hypothyroid and $40 \%$ euthyroid at 10 years when given a "low dose" protocol. ${ }^{17}$ But when a "moderate dose" protocol is used 93\% require thyroxine replacement at one to five years. ${ }^{18}$ The risk of early thyroid failure after the higher dose is thought by many to be more convenient in terms of follow up than delayed failure. The long term safety of RAI therapy has been described in well designed and conducted cohort studies. ${ }^{19}$ ATD may be required before and after RAI in severe thyrotoxicosis (particularly in the elderly population) with a drug free period of three to seven days on either side of RAI therapy. However, many particularly in the USA do not use ATD routinely in preparation for RAI therapy.

\section{Surgery}

Surgery is reserved for patients with GD who are unwilling/ unable to have RAI (for example, those with young children), those who express a cosmetic preference, and for those with suspicious goitres. Rarely, surgery may be needed in those who develop severe side effects to ATD in whom RAI is contraindicated (mid-pregnancy and lactation) or undesirable. ATD may be needed before surgery particularly in the elderly and in those with severe disease, mainly to avoid the risk of precipitating a "thyroid storm" with its attendant morbidity and mortality.

\section{THYROID EYE DISEASE (TED)}

Up to $5 \%$ with TED require active intervention for sight threatening disease. The rest may require symptom relief with artificial tears, sleeping propped up, and protection from dust. Stopping smoking is essential. Those requiring active treatment may need corticosteroids (for soft tissue, recent extraocular and optic nerve involvement; given intravenously for greater effect), orbital decompression surgery or radiotherapy alone or in combination. Corrective surgery is undertaken when thyroid eye disease is quiescent. Improvement in function, appearance, and vision are variable. ${ }^{20}$ Antioxidants, cytokine antagonists, and somatostatin analogues are experimental. ${ }^{21}$

Controversy still surrounds the effects of RAI on TED. Several studies have shown a small but definite deterioration of TED with its use, prevented by concomitant corticosteroid cover-prednisolone $30-40 \mathrm{mg} /$ day for one month after RAI therapy with dose tapering thereafter. ${ }^{22}$

\section{NODULAR THYROID DISEASE, TOXIC ADENOMA (TA), AND TOXIC MULTINODULAR GOITRE (TMNG)}

The thyroid may over a variable period develop single or multiple nodules that may hyperfunction (TA and TMNG respectively) or be non-functioning. The exclusion of malignancy is important in some and may require fine needle aspiration biopsy (FNAB), ultrasound and nuclear imaging, and biochemical tests. ${ }^{23}$ Nodules that are very firm/ hard, growing rapidly, attached to surrounding structures, or associated with regional lymph nodes, distant metastases, or vocal cord paralysis are highly suspicious and should be biopsied. ${ }^{24}$ Lifelong follow up is recommended, ${ }^{24}$ although a range of views exists about the investigation and management of thyroid nodules. ${ }^{25-27}$ Molecular genetics may increase the sensitivity of FNAB (for example, detecting RET/PTC gene rearrangements in papillary cancers). Management may include the following:

(1) Suppressive thyroxine therapy for benign disease-has limited usefulness and is contraindicated in patients with suppressed TSH. At recommended dose may cause cardiac and bone side effects

(2) RAI therapy-preferred method of treatment for TA and TMNG. Large non-functioning goitres may diminish in size.

(3) Surgery-reserved for large goitres and those causing pressure symptoms, nodules with malignant, follicular, or indeterminate cytology at FNAB and for patient preference.

(4) A significant proportion of those who undergo surgery or RAI therapy will be hypothyroid eventually and will require thyroxine therapy as described above.

Detailed management of malignant thyroid nodules is not within the scope of this review. 


\section{DRUG INDUCED THYROID DISEASE}

Several commonly used drugs may cause thyroid dysfunction. We discuss briefly amiodarone, interferon, and lithium induced thyroid dysfunction here.

\section{Amiodarone}

Amiodarone may cause both hypothyroidism (AIH) and thyrotoxicosis (AITl and 2) (table 2). ${ }^{28}$

Very few with AIH are symptomatic. Moderately raised TSH (up to $20 \mathrm{mU} / \mathrm{l}$ ) and high normal or high free T4 shows subclinical hypothyroidism-most with raised TSH and high normal free T4 who are TPOAb+ve benefit from thyroxine as they progress to clinical hypothyroidism. Higher TSH activity and low normal free T4 shows significant hypothyroidism requiring treatment.

AIT is confirmed by a combination of high free T3 and undetectable or significantly low TSH activity. However, normal free $\mathrm{T} 3( \pm$ high free $\mathrm{T} 4)$ combined with the above TSH abnormalities suggest subclinical hyperthyroidism only. High dose CMZ/PTU is given for 6-12 weeks and withdrawal of ATD attempted. Corticosteroids need to be added to CMZ/ PTU when tests are inconclusive, in mixed forms of AIT or response to ATD is inadequate. For recurrences, RAI therapy may be tried (after RAI uptake is established). Some require indefinite CMZ/PTU therapy. Life threatening AIT requires surgery if other therapy fails. Although continuation of amiodarone may have advantages partly because of its cardioprotective antiadrenergic properties, ${ }^{29}$ withdrawal should be attempted whenever possible.

\section{Lithium}

About 5\% develop hypothyroidism while taking lithium and benefit from thyroxine therapy. ${ }^{30}$

\section{Interferon alfa}

An early destructive thyroiditis may cause transient mild thyrotoxicosis (TRAb-ve, low/absent RAI uptake, reduced flow on colour Doppler scanning) and may require symptomatic treatment with $\beta$ blockers. Some may develop late hypothyroidism. An exacerbation of GD (TRAb+ve, high RAI uptake) requires standard therapy as described earlier. Those with previous autoimmunity may develop hypothyroidism requiring thyroxine therapy (specially those receiving combination therapy with ribavarin). ${ }^{31}$

\section{PREGNANCY AND THYROID DYSFUNCTION}

The changing immune environment and the requirements of a growing fetus make pregnancy and the postpartum period complicated and important. There is good evidence for the need for strict control of thyroid homoeostasis particularly during organogenesis. ${ }^{32}$

\section{Hyperthyroidism}

There are several causes of hyperthyroidism during pregnancy. GD and gestational transient thyrotoxicosis (GTT) associated with hyperemesis gravidarum are the commonest. The need for treatment of thyrotoxicosis in pregnancy is based on adverse maternal (placental abruption, preterm delivery, heart failure, thyroid storm, etc) and fetal (low birth weight, growth retardation, neonatal hyperthyroidism, stillbirth, and fetal death, etc) outcomes.

Human chorionic gonadotrophin (HCG) is structurally related to TSH and high HCG activity in women with hyperemesis causes a transient sometimes moderately severe thyrotoxicosis. These women are TRAb-ve and may require short term ATD therapy. ${ }^{33}$

GD in pregnancy is managed as follows:

(1) PTU is preferred because of a lack of teratogenesis (aplasia cutis and embryopathy are extremely rarely seen with other ATDs $)^{34}$

(2) Patients with previous RAI or surgically treated GD who are euthyroid $( \pm$ LT4) should have their TRAb checked early in pregnancy and at 36 weeks of gestation. Early high TRAB titres or detectable TRAb later are predictive of fetal hyperthyroidism. Joint obstetric, paediatric, and endocrine input is essential. ${ }^{35}$

(3) Patients who are thyrotoxic during pregnancy should be given PTU $100-150 \mathrm{mg}$ thrice daily until they are euthyroid and dose reduced rapidly until free $\mathrm{T} 4$ is at the upper limit of the reference range. This dose should be continued through pregnancy and labour while monitoring thyroid function every four to six weeks. It may be possible to stop PTU in the third trimester in some. TRAb should be checked and the patient jointly managed as described above. A postpartum exacerbation may occur in some. ${ }^{36}$

(4) Fetal thyrotoxicosis (tachycardia and growth retardation) occurs through transplacental transfer of TRAb. Fetal monitoring during pregnancy and TRAb checks as described above are therefore important. Transient neonatal thyrotoxicosis may require $\beta$ blockers or rarely ATD in the infant. ${ }^{36}$

(5) Surgery should be avoided unless poor control resulting from poor compliance or side effects poses a risk to mother and child. It should be undertaken in the second trimester if possible.

\section{Hypothyroidism}

There is good evidence to suggest that maternal and fetal wellbeing are compromised by hypothyroxinaemia, and subclinical and overt hypothyroidism. In addition to previously known effects, recent interest has centred on

Table 2 Amiodarone induced thyrotoxicosis (AIT)

\begin{tabular}{lll}
\hline & AIT 1 & AIT2 \\
\hline Thyroid & Goitre + & Normal \\
Ultrasound imaging & MNG/diffuse goitre & Normal \\
Colour flow Doppler scan & Normal or increased flow & Decreased flow \\
Thyroid antibodies & Absent/present & Absent generally \\
Interleukin 6 & Normal or high & Very high \\
RAl uptake & Low or normal & Very low/absent \\
Preferred treatment & Carbimazole/PTU Potassium & Glucocorticoids \\
& perchlorate & \\
\hline
\end{tabular}

AIT-more common in iodine deficient $(12 \%)$ compared with sufficient areas $(2 \%)$. AIT1 results from iodine excess and AIT2 is a destructive thyroiditis. Clinically a combination of AIT1 and 2 often occurs. AlH-amiodarone induced hypothyroidism is more common in iodine replete (13\%) compared with iodine deficient areas (6\%). Hormone synthesis is reduced particularly in TPOAb positive patients. 
impaired neurointellectual development of the child. ${ }^{32}$ Adequate transfer of thyroxine is vital at the stage of organogenesis in the first trimester. When pregnancy is confirmed, the dose of LT4 should be increased by 50-100 $\mu \mathrm{g}$ / day in previously hypothyroid patients. In women without previous hypothyroidism, most authorities would recommend immediate full replacement LT4 therapy in those with (a) clearly abnormal thyroid function-that is, TSH $>4 \mathrm{mU} / \mathrm{l}$ or free T4 below the reference range for gestational age irrespective of thyroid autoimmune status, (b) thyroid autoimmunity (TPOAb+ve) and TSH between $2-4 \mathrm{mU} / \mathrm{l}$ or free $\mathrm{T} 4$ in the low normal/low range. However, more prospective RCTs are needed for validating such a management strategy. The LT4 dose increase that occurs as pregnancy progresses should be individually tailored and guided by frequent thyroid tests and reduced after delivery.

\section{Postpartum thyroid dysfunction (PPTD)}

Nearly half of the $10 \%-11 \%$ of women who are TPOAb+ve in early gestation develop PPTD. Although a largely self limiting disease, about 30\% who are TPOAb+ve and develop PPTD are permanently hypothyroid within the first year and nearly half are clinically or subclinically hypothyroid at long term review. ${ }^{37}$ Symptomatic treatment with $\beta$ block suffices for most in the early thyrotoxic phase. But a majority who develop hypothyroidism later on require either temporary (with trial of withdrawal in 9-12 months) or permanent LT4 therapy. Although no screening strategy is in place, high risk women such as those with previous PPTD (70\% have a recurrence) and type 1 diabetic patients (25\% develop PPTD) may be targeted for early screening with TPOAb. ${ }^{38}$

\section{SUBCLINICAL THYROID DISEASE}

The increasingly frequent diagnosis of subclinical thyroid dysfunction is probably the result of the wide use of thyroid testing in clinical practice. Such people are largely asymptomatic but have adverse outcomes for vascular and bone disease. There are no clear guidelines, but some principles guiding management have emerged.

\section{Subclinical hypothyroidism (SH)}

SH (TSH $>4.5 \mathrm{mU} / \mathrm{l}$ with normal free $\mathrm{T} 4$ and $\mathrm{T} 3$ ) is seen in $7.5 \%$ and $2.8 \%$ women and men respectively and is more common in the elderly population (17 compared with 7\% in women and men $>65$ respectively). TSH returns to normal in about $5 \%$ in the first year and repeating thyroid tests in 412 weeks is therefore important before treatment is given. Patients with TSH $>10 \mathrm{mU} / \mathrm{l}$ should be treated with LT4. ${ }^{39}$ The evidence for outcomes is more compelling in this group than in those with TSH $<10 \mathrm{mU} / \mathrm{l}$. Pregnancy and anticipation of pregnancy are indications for LT4 treatment at even lower TSH activity (see above). In subjects with significant symptoms a trial of therapy may be given for three to six months-25\%-30\% improve. Patients with TSH between 4.5 and $10 \mathrm{mU} / \mathrm{l}$ should be monitored annually ${ }^{39}$ and individually assessed for a trial of thyroxine therapy, particularly if they are TPOAb positive. ${ }^{40}$ There is recent evidence to suggest that high TSH activity does not cause adverse effects in the very elderly ${ }^{41}$ Treatment does not change the natural history of $\mathrm{SH}$.

\section{Subclinical hyperthyroidism (SHyper)}

SHyper is common and occurs during exogenous LT4 therapy (in those given suppressive doses for cancer and benign nodular disease and replacement doses for hypothyroidism) and in endogenous thyroid disease (for example, GD, TA, and TMNG). Reversible and preventable effects may occur on quality of life, cardiovascular function and outcome, and bone density. ${ }^{42}$ Careful monitoring of target TSH activity during LT4 therapy is important. In elderly patients who require long term suppressive therapy, prophylactic $\beta$ blockers and bisphosphanates should be considered. Standard therapy as for overt disease (ATD, RAI, or surgery) is recommended by some for endogenous SHyper in elderly patients whose TSH is undetectable or in those whose TSH is $<0.1 \mathrm{mU} / \mathrm{l}$ and who have a high risk of vascular disease or decreased bone density. Clear evidence and guidelines are needed in this area.

\section{THYROIDITIS}

Acute thyroiditis may complicate bacterial, fungal, and parasitic infections. FNAB provides microbiological proof guiding therapy.

Subacute (De Quervain's) thyroiditis is self limiting, commoner in women, and associated with a high erythrocyte sedimentation rate and low RAI uptake. The initial thyrotoxic phase may require $\beta$ blockers and the late hypothyroid phase LT4 therapy. Pain relief is obtained from aspirin, nonsteroidal analgesics, and the occasional use of corticosteroids.

Subacute lymphocytic thyroiditis is painless but is managed as above.

Reidel's thyroiditis occurs in middle aged or elderly women who are usually euthyroid. Surgical biopsy aids diagnosis. Wedge resection of the isthmus, corticosteroids, and tamoxifen may be tried.

\section{THYROID HORMONE RESISTANCE SYNDROMES}

These uncommon dominantly inherited disorders are largely asymptomatic and are mainly attributable to thyroid hormone $\beta$ receptor gene mutations. Although subjects with generalised resistance (GRTH) are clinically hypo/euthyroid (compensating with raised thyroid hormone production) and those with pituitary resistance (PRTH) may be hyperthyroid, there may be no clear clinical distinction. Inappropriately raised TSH activity (that is, non-suppressed in the presence of raised free T4) is characteristic and needs to be differentiated from the rare TSH secreting pituitary tumour. Thyroid antibodies are absent.

Most people need no treatment. Judicious use of thyroxine may be needed in others. Patients with thyrotoxicosis may benefit from $\beta$ blockers-dopamine agonists, somatostatin, and TRIAC have produced mixed results.

\section{NON-THYROIDAL ILLNESS SYNDROME (NTIS)}

Patients with NTIS have abnormalities in thyroid function that may cause diagnostic confusion. Usually total T3 is subnormal but total T4 may also be low and in some cases TSH may be high. This pattern is seen in critically ill patients and has a poor prognosis. ${ }^{43}$ The free T4 may be normal. Several factors may cause these abnormalities. Impaired peripheral T4 to T3 conversion, changed binding of thyroid hormones (inhibitors of binding may be present), and cytokines may play a part.

Are these patients hypothyroid? If the TSH is $>20 \mathrm{mU} / \mathrm{l}$ they probably are and LT4 therapy may be tried. However, most are euthyroid and most trials of LT4 therapy in NTIS have not been efficacious. T3 therapy may be of limited benefit in patients having cardiothoracic procedures. The high frequency of NTIS in acutely ill patients in hospital makes routine thyroid testing in these subjects of little use.

\section{THE FUTURE}

Great advances have been made in eliminating iodine deficiency during the past two decades but the condition is still widely seen. Continued vigilance is necessary but an end to this treatable problem should be our goal.

Although considerable advances have been made in our understanding of the immune system, we still do not understand thyroid autoimmunity completely. Eventual 
therapy of Graves' and Hashimoto's disease pharmacoimmunologically may be possible and may be important in Graves' orbitopathy where no such treatment is available now.

Molecular biology has been used to study thyroid physiology (action of thyroid hormones at a cellular level) and has resulted in the production of hormone analogues that may be useful in obesity and other conditions. These represent good examples of scientific excellence in collaboration with the pharmaceutical industry.

The effects of thyroid hormones in the developing brain have opened up a new area of investigation. The delineation of regional specificity for thyroid hormone receptors at different gestational ages and in adult life is exciting and offers potential for new developments. There is evidence that maternal hormone deficiency results in impaired neurodevelopment. The value of screening for thyroid dysfunction in early gestation remains to be determined.

Although thyroid cancer accounts for only $1 \%$ of all malignancies, it is the commonest endocrine tumour. Anaplastic cancer is a death sentence in the elderly person. Again, molecular biology has provided much knowledge in all tumour types and may result in targeted gene therapy for these tumours.

Advances in thyroidology resulting in improved clinical care of our patients have been derived from a close relationship between scientists, clinical scientists, and clinicians. It is essential to maintain these links in the future for our ultimate understanding of these diseases and their rational therapy.

\section{MULIPLE CHOICE QUESTIONS (TRUE (T)/FALSE (F); ANSWERS AFTER THE REFERENCES)}

1. In Hashimoto's thyroiditis

(A) Some hypothyroid patients will become euthyroid without treatment

(B) Combination therapy with thyroxine and liothyronine is recommended for initial therapy

(C) Persistent TSH increase in a compliant patient receiving standard thyroxine therapy, may be attributable to concomitant drug therapy

(D) TSH should be suppressed in subjects receiving adequate thyroxine therapy

(E) Pregnant subjects do not usually need to increase their dose of thyroxine

2. In the management of Graves' disease

(A) Recurrences are commonest after the second year after stopping treatment

(B) TSH normalises even before free T4 becomes normal

(C) Periodic neutrophil counts may be used to predict thionamide induced agranulocytosis

(D) Propylthiouracil may cause hepatitis with a poor prognosis

(E) Radioiodine therapy is the treatment of choice in the elderly patient

3. Amiodarone

(A) Causes symptomatic hypothyroidism in the majority

(B) Induced thyrotoxicosis type 2 responds well to thionamides

(C) May be continued in those developing thyrotoxicosis

(D) Induced hypothyroidism is more common in iodine sufficient areas

(E) Induced thyrotoxicosis is characterised by normal free $\mathrm{T} 3$ and low or undetectable TSH activity
4. In pregnancy

(A) Thyrotoxicosis attributable to hyperemesis gravidarum may require thionamide therapy

(B) Carbimazole is preferred to propylthiouracil because of lack of teratogenicity

(C) Early high or late thyrotrophin receptor antibody activity predicts fetal hyperthyroidism

(D) TSH activity of $8 \mathrm{mU} / \mathrm{l}$ in the absence of TPO antibody does not point to the need for thyroxine treatment

(E) Increased thyroxine requirements in hypothyroid patients will persist in the postpartum period

5. Subclinical thyroid dysfunction

(A) Is uncommon in the elderly person

(B) Causing undetectable TSH activity may increase risk of bone and vascular disease

(C) Causing a TSH activity of $>5 \mathrm{mU} / \mathrm{l}$ always requires thyroxine therapy

(D) Is more common in men

(E) With raised TSH activity should be monitored until the third trimester in pregnant women before treatment is started

\section{Authors' affiliations}

L D K E Premawardhana, Department of Medicine, Caerphilly Miners'

Hospital, Caerphilly, UK

J H Lazarus, Department of Medicine, University Hospital of Wales, Cardiff, UK

Funding: none.

Conflicts of interest: none.

\section{REFERENCES}

1 Weetman AP. Autoimmune thyroid disease; propagation and progression. Eur J Endocrinol 2003; 148: 1-9.

2 Simmonds MJ, Gough SCL. Genetic insights into disease mechanisms of autoimmunity. Br Med Bull 2005;71:93-113.

3 Tunbridge WM, Evered DC, Hall R, et al. The spectrum of thyroid disease in a community; the Whickham survey. Clin Endocrinol (Oxf) 1977;7:481-93.

4 Vanderpump MP, Tunbridge WM, French JM, et al. The incidence of thyroid disorders in the community; a twenty year follow up of the Whickham survey. Clin Endocrinol (Oxf) 1995;43:55-68.

5 Flynn RWV, MacDonald TM, Morris TM, et al. The thyroid epidemiology, audit and research study: thyroid dysfunction in the general population. J Clin Endocrinol Metab 2004;89:3879-83.

6 Bulow Pedersen I, Knudsen N, Jorgensen T, et al. Large differences in incidences of overt hyper and hypothyroidism associated with a small difference in iodine intake: a prospective comparative register based population survey. J Clin Endocrinol Metab 2002;87:4462-9.

7 Sato K, Okamura K, Hirata T, et al. Immunological and chemical types of reversible hypothyroidism; clinical characteristics and long-term prognosis. Clin Endocrinol 1996;45:519-24.

8 Baloch Z, Carayon P, Conte-Devolx B, et al. Laboratory medicine practice guidelines; Laboratory support for the diagnosis and monitoring of thyroid disease. Thyroid 2003;13:3-126.

9 Walsh JP, Shiels L, Lim EM, et al. Combined levothyroxine/liothyronine treatment does not improve well being, quality of life, or cognitive function compared to thyroxine alone: a randomised controlled trial in patients with primary hypothyroidism. J Clin Endocinol Metab 2003;88:4543-50.

10 The American Thyroid Association, The Endocrine Society, American Association of Clinical Endocrinologists. Joint statement on the US Food and Drug Administration's decision regarding bioequivalence of levothyroxine sodium. Thyroid 2004; 14:486.

11 Cooper DS. Drug therapy; antithyroid drugs. N Engl J Med 2005;352:905-17.

12 Weetman AP. Graves' disease. N Engl J Med 2000;347:1232-48.

13 Weetman AP. Graves' hyperthyroidism; how long should antithyroid drug therapy be continued to achieve remission? Nature Clin Pract Endocrinol Metab 2006;2:2-3.

14 Abraham P, Avenell A, Park AM, et al. A systematic review of drug therapy for Graves' hyperthyroidism. Eur J Endocrinol 2005; 153:489-98.

15 Schott M, Morgenthaler NG, Fritzen R, et al. Levels of autoantibodies against human TSH receptor predict relapse of hyperthyroidism in Graves' disease. Horm Metab Res 2004;36:92-6. 
16 Nedrebo BG, Holm Pl, Uhlving S, et al. Predictors of outcome and comparison of different drug regimes for the prevention of relapse of patients with Graves' disease. Eur J Endocrinol 2002;147:583-9.

17 Rondebush CP, Hoye KE, De Groot L. Compensated low dose 1131 therapy in Graves' disease. Ann Intern Med 1977;17:441-7.

18 Berg G, Michande A, Holmberg E, et al. Clinical outcome of RAl treatment for hyperthyroidism. J Intern Med 1996;239:168-71.

19 Franklyn JA, Maisonneuve P, Sheppard MC, et al. Cancer incidence and mortality after RAl therapy for hyperthyroidism - a population based cohort study. Lancet 1999;353:2111-15.

20 Kraassas GE, Wiersinga W. Thyroid eye disease; current concepts and the EUGOGO perspective. Thyroid International 2005;4:3-21.

21 Cawood T, Moriarty P, O'Shea D. Recent developments in thyroid eye disease. BMJ 2004:329:385-90.

22 Bartalena L, Marcocci C, Pincera A. Graves' ophthalmopathy; a preventable disease? Eur J Endocrinol 2002;146:457-61.

23 Hegedus L, Bonnema SJ, Bennedbaek FN. Management of simple nodular goitre; current status and future perspectives. Endocr Rev 2003;24:102-32.

24 Hegedus L. The thyroid nodule. N Engl J Med 2004;351:1764-71.

25 Singer PA, Cooper DS, Daniels GH, et al. Treatment guidelines for patients with thyroid nodules and well-differentiated thyroid cancer. Arch Intern Med 1996; 156:2165-72

26 Feld S, Garcia M, Baskin HJ et al. AACE clinical practice guidelines for the diagnosis and management of thyroid nodules. Enodcr Pract 1996;2:78-84.

27 Castro MR, Gharib H. Continuing controversies in the management of thyroid nodules. Ann Intern Med 2005; 142:926-31.

28 Martino E, Bartalena L, Bogazzi F, et al. The effects of amiodarone on the thyroid. Endocr Rev $2001 ; 22: 240-54$.

29 Osman F, Franklyn JA, Sheppard MC, et al. Successful treatment of amiodarone induced thyrotoxicosis. Circulation 2002;105:1275-7.

30 Kirov G, Tredget J, John R, et al. A cross-sectional and a prospective study of thyroid disorders in lithium treated patients. J Affect Disord 2005:87:313-17.

31 Carella C, Mazziotti G, Amato G, et al. Interferon alpha related thyroid disease; pathophysiological, epidemiological and clinical aspects. J Clin Endocrinol Metab 2004;89:3656-61.

32 Haddow JE, Palomaki GE, Allen WC, et al. Maternal thyroid deficiency and subsequent neuropsychological development of the child. N Engl J Med 1999;341:549-55.
33 Kimura M, Amino N, Tamaki $\mathrm{H}$, et al. Gestational thyrotoxicosis and hyperemesis gravidarum; possible role for hCG with higher stimulating activity. Clin Endocrinol (Oxf) 1993;38:345-50.

34 Mandel SJ, Cooper DS. The use of antithyroid drugs in pregnancy and lactation. J Clin Endocrinol Metab 2001;86:2354-9.

35 Laurberg P, Nygaard B, Glinoer D, et al. Guidelines for TSH receptor antibody measurements in pregnancy; results of an evidence-based symposium organised by the European Thyroid Association. Eur J Endocrinol 1998; 139:584-6.

36 Lazarus JH. Thyroid disorders associated with pregnancy. Treat Endocrinol 2005; 4:31-41.

37 Premawardhana LDKE, Parkes $A B$, Ammari $F$, et al. Postpartum thyroiditis and long term thyroid status; influence of thyroid peroxidase antibodies and ultrasound echogenicity. J Clin Endocrinol Metab 2000;85:71-5.

38 Lazarus JH, Premawardhana LDKE. Screening for thyroid disease in pregnancy. J Clin Pathol 2005;58:449-52.

39 Col NF, Surks MI, Daniel GH. Subclinical thyroid disease. JAMA 2004;291:239-43.

40 Gharib H, Tuttle M, Baskin J, et al. Consensus statement: subclinical thyroid dysfunction: A joint statement on the management from the American Association of Clinical Endocrinologists, the American Thyroid Association and the Endocrine Society. J Clin Endocrinol Metab 2005;90:581-6.

41 Gussekloo J, van Exel E, de Crein AJ, et al. Thyroid status, disability and cognitive function and survival in old age. JAMA 2004;292:2591-9.

42 Biondi B, Palmieri EA, Klain M, et al. Subclinical hyperthyroidism; clinical features and treatment options. Eur J Endocrinol 2005;152:1-9.

43 Chopra I. Euthyroid sick syndrome: Is it a misnomer? J Clin Endocrinol Metab 1997;82:329-34.

\section{ANSWERS}

1. (A) T, (B) F, (C) T, (D) F, (E) F; 2. (A) F, (B) F, (C) F, (D)

T, (E) T; 3. (A) F, (B) F, (C) T, (D) T, (E) F; 4, (A) T, (B) F, (C)

T, (D) F, (E) F; 5. (A) F, (B) T, (C) F, (D) F, (E) F. 